\title{
Evaluation of therapeutic effects of FAK inhibition in murine models of atherosclerosis
}

\author{
Takeshi Yamaura', Tatsuhiko Kasaoka ${ }^{1}$, Naoko lijima', Masaaki Kimura ${ }^{1}$ and Shinji Hatakeyama ${ }^{2}$ [0]
}

\begin{abstract}
Objective: Therapeutic effects of focal adhesion kinase (FAK) inhibition using a small molecule inhibitor was evaluated in apolipoprotein $\mathrm{E}(\mathrm{apoE})$ knockout $(\mathrm{KO})$ and low-density lipoprotein receptor (LDLr) KO mouse atherosclerosis models.

Results: The prevention trial consisted of an 8-week treatment with an FAK inhibitor concurrent treatment with a high fat (HF)/high cholesterol (HC) diet. The intervention trial consisted of 6- and 8-week treatment after 6- and 8-week pre-loading, respectively, of a HF/HC diet in apoE KO and LDLr KO mice, respectively. The inhibitor was admixed with a HF/HC diet and mice were given free access to the admixture. The FAK inhibitor exhibited marked inhibition against the development of the atherosclerosis in both of prevention and intervention trials at a dose of $0.03 \%$ without showing any remarkable toxic properties in biochemical examinations. These results indicated that FAK inhibition might be a possible candidate for novel therapeutic targets against atherosclerosis.
\end{abstract}

Keywords: Atherosclerosis, Focal adhesion kinase, apoE KO, LDLr KO

\section{Introduction}

Focal adhesion kinase (FAK) is a non-receptor cytoplasmic tyrosine kinase that regulates multiple cell functions [1]. Activation of integrins and the growth factor receptors result in FAK autophosphorylation at Y397 and the presentation of suitable binding sites for proteins containing either Src homology 2 domain or phosphotyrosine binding domains. Association of FAK with PI3-kinase is thought to be necessary for FAK signaling pathway because phosphorylation of FAK on Y397 is required for cell proliferation, apoptosis and migration [2]. It is well known that elevated expression levels of FAK have been detected in various tumor samples and are closely correlated with invasive potential [3]. FAK is considered to be involved in inflammatory pathways such as leukocyte rolling, attachment and motility as well as migration of smooth muscle cells and endothelial cells $[4,5]$, but the roles of FAK in the development of atherosclerosis still remain to be clarified. Here we report the effect of FAK

\footnotetext{
*Correspondence: shinji.hatakeyama@novartis.com

${ }^{2}$ Novartis Pharma AG, Basel, Switzerland

Full list of author information is available at the end of the article
}

inhibition on the atherosclerosis in the spontaneous model using apoE KO and LDLr KO mice.

\section{Main text \\ Methods}

The mutant male mice, apoE KO (B6.129P2-Apoe $e^{\text {tm1Unc } / J) ~}$ and LDLr KO (B6.129S7-Ldlr $\left.{ }^{\text {tm1Her }} / \mathrm{J}\right)$ at the age of 7 weeks, were purchased from Charles River Laboratories, and acclimated to the facility for 7 days. The mice were housed at $25{ }^{\circ} \mathrm{C}$ with a 12:12 h light-dark cycle, and food and water were provided ad libitum. The HF/ HC diet D12108 containing 1.25\% cholesterol and 20\% fat was purchased from Research Diets. A selective small molecule FAK inhibitor Compound 12 (Additional file 1: Figure S1; a 2-phenylamino-pyrimidin-4-ylamino-2,3-dihydro-isoindol-1-one derivative) was administrated as food admixture at a concentration of $0.03 \%$. Compound 12 has an $\mathrm{IC}_{50}$ of $6 \mathrm{nM}$ on FAK and no activity on insulin receptor kinase, insulin-like growth factor 1 receptor kinase, cyclin-dependent kinase 1 and Src (Additional file 2: Table S1) as described by Kawahara et al. [6]).

To assess the therapeutic effect of FAK inhibition, the following three different designs were applied: prevention 
(1) and therapeutic intervention (2) in apoE $\mathrm{KO}$ mice, and therapeutic intervention (3) in LDLr KO mice. The prevention trial consisted of 8-week concurrent treatment with the HF/HC diet following 1 week of pre-loading of the HF/HC diet (Additional file 3: Figure S2A). The therapeutic intervention trial consisted of 6- and 8-week treatment after 6- and 8-week pre-loading of the $\mathrm{HF} / \mathrm{HC}$ diet in apoE KO and LDLr KO mice, respectively (Additional file 3: Figure S2B). Body weight and food intake were measured once weekly. The mice were euthanized under terminal inhalation anesthesia with isoflurane followed by blood collection from abdominal vein.

Atherosclerotic lesion area was evaluated in the aorta by en face analysis (Additional file 4: Figure S3A) and in aortic root by cross-sectional analysis (Additional file 4: Figure S3B). The aorta was subjected to Sudan IV staining, and total area of the plaque lesion (TAOL) and area of whole aorta from arch without the region of brachiocephalic artery to common iliac artery (AOWA) were measured using the Definiens imaging software. Area of lesion (\%) was calculated by the following formula; Area of lesion $(\%)=\mathrm{TAOL} / \mathrm{AOWA} \times 100$. After en face analysis, the aorta was washed and then blotted dry, weighed, minced, and total lipid was extracted with chloroform/ methanol. Total and free cholesterol levels in the aortic extracts were determined with an enzymatic colorimetric assay (Wako Pure Chemical Industries Ltd.). Cross sections were cut at $5 \mu \mathrm{m}$ thickness and 6 serial sections were collected every $50 \mu \mathrm{m}$ along the aortic root. Lesion area analysis was made on the cross sections subjected to hematoxylin and eosin (HE) staining. Three representative sections derived from 3 segments were quantified per mouse. Lesion area values from the 3 sections were summed up. Histological features using serial cross sections were subjected to staining for EvG and anti Mac-3 antigen. Clinical biochemistry markers in serum was evaluated using DRICHEM 7000 (FUJIFILM Medical, Japan). Statistical comparison of control and compound 12-treated groups was carried out with a Mann-Whitney test (two-tailed) using GraphPad Prism.

\section{Results and discussion}

The effect of a selective FAK inhibitor, Compound 12 [6] was evaluated in apoE KO and LDLr KO mouse spontaneous models of atherosclerosis in 2 different designs, prevention and therapeutic intervention trials. Compound 12 was generally well tolerated and there were no remarkable changes in body weight and food intake in 3 trials: prevention in apoE KO mice (Fig. 1a, b), therapeutic intervention in apoE KO mice (Fig. 2a, b) and in LDLr KO mice (Fig. 3a, b).

Compound 12 showed a marked inhibitory effect on the development of the atherosclerosis in the prevention trial using apoE $\mathrm{KO}$ mice and the intervention trials using apoE KO and LDLr KO mice. In the prevention trial in apoE KO mice, Compound 12 significantly reduced the lesion area of the aorta by $41.2 \%$ (Fig. 1c; $8.92 \%$ in Control and 5.24\% in Compound 12), and also significantly reduced the cholesterol content (Fig. 1d) and lesion area of aortic root (Fig. 1e). A representative picture of en face analysis of the aorta stained withF Sudan IV is presented in Fig. 1f. Histological features using serial cross sections with HE, EvG and anti Mac-3 are presented in Fig. 1g (higher magnifications in lower panels). Interestingly, in atherosclerotic plaques from the control group, cholesterol crystals, fibrous and lipid core regions were observed displaying histological features similar to those seen in human, while in atherosclerotic plaques from the Compound 12-treated group, foam cell rich, reduced fibrous and lipid core regions were observed.

In the therapeutic intervention trials in apoE $\mathrm{KO}$ and LDLr KO mice, Compound 12 significantly reduced the lesion area of the aorta by $40.9 \%$ in apoE $\mathrm{KO}$ mice (Fig. 2c; $8.03 \%$ in Control at 12 weeks and $4.75 \%$ in Compound 12 normalized to Control at 6 weeks) and by $30.9 \%$ in LDLr KO mice (Fig. 3c; $8.51 \%$ in Control at 16 weeks and 5.83\% in Compound 12 normalized to Control at 8 weeks), and also significantly reduced the cholesterol content (Figs. 2d, 3d). The lesion area of aortic root appeared to be reduced in apoE KO mice (Fig. 2e).

There were some changes in clinical biochemistry markers (Additional file 5: Table S2): a significant or a trend of reduction in ALT, LDH and T-Chol may suggest a protective effect of FAK inhibition on organs under the $\mathrm{HF} / \mathrm{HC}$ condition, and in contrary, a significant or a trend of increase in ALP, TG and CRE could indicate ontarget safety flags. The mechanism behind T-Chol lowering is unclear.

The precise mechanism of how FAK inhibition could prevent or inhibit atherosclerosis remains unclear although a pathological role of FAK in atherosclerosis has been reported, such as its activation by remnant lipoproteins through RhoA and s1-integrin [7], by angiotensin II through Akt-mTOR-NF- $k B$ signaling pathway [8], and by oxidized LDL through $\mathrm{p} 90$ ribosomal $\mathrm{S} 6$ kinase family proteins [9]. These results would suggest that FAK might play an important role in the development of atherosclerosis and FAK inhibition might be a possible candidate for novel therapeutic targets for the treatment of atherosclerosis.

\section{Limitations}

In the experiments described in this report, we only evaluated the efficacy of the FAK inhibitor and did not assess any biomarkers related to FAK activation or inhibition, and atherosclerosis. Further assessment in various 
a

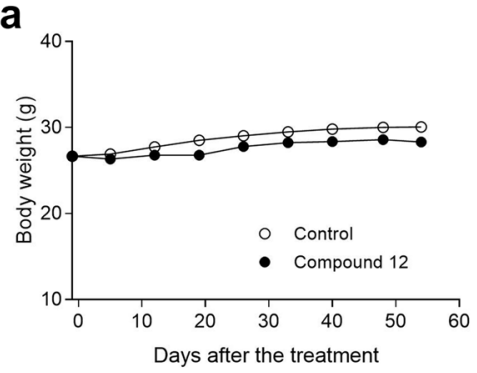

b

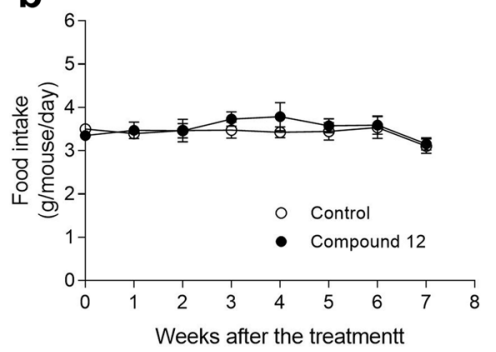

C

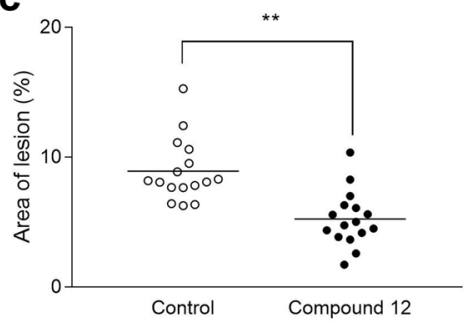

d

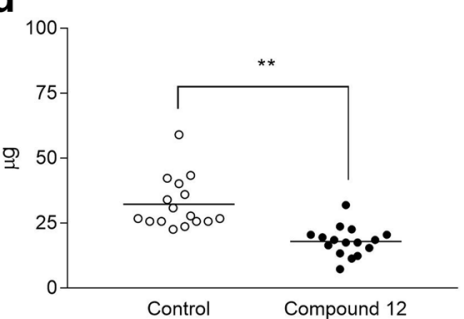

e

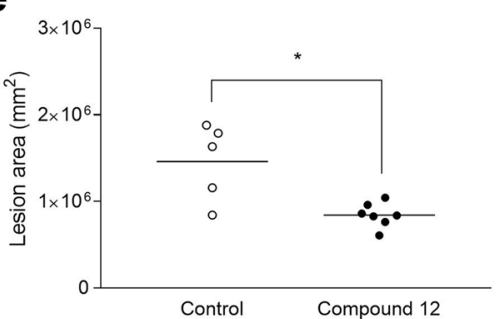

f

Control Compound 12

\section{g}

HE

EvG
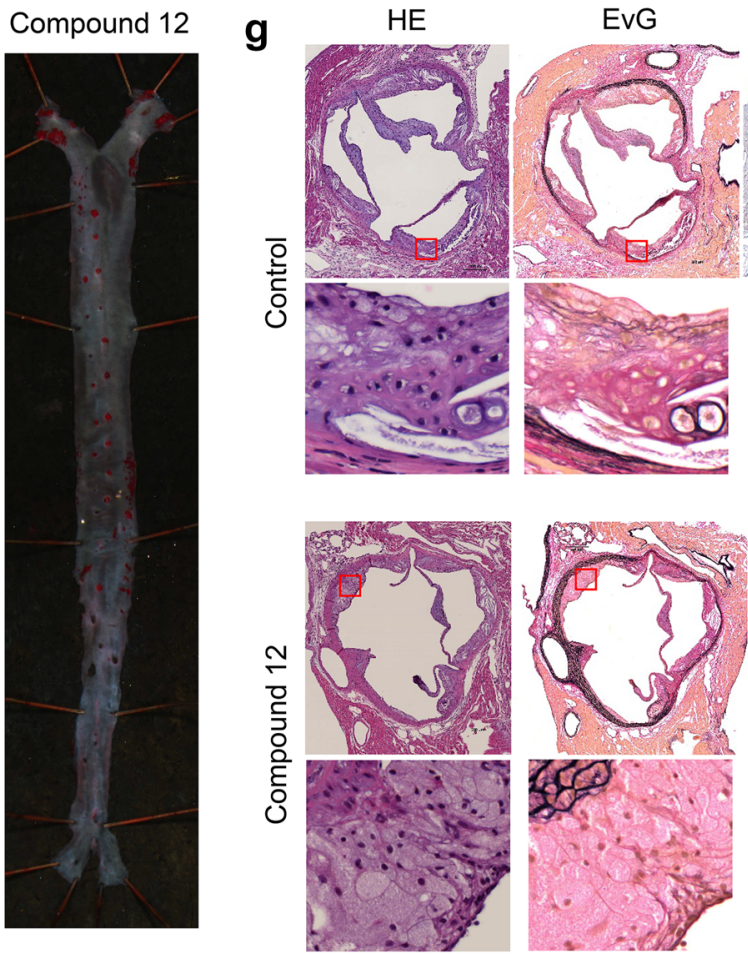

Mac-3

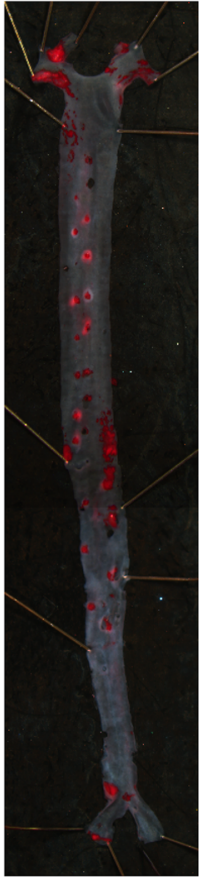

ठำ
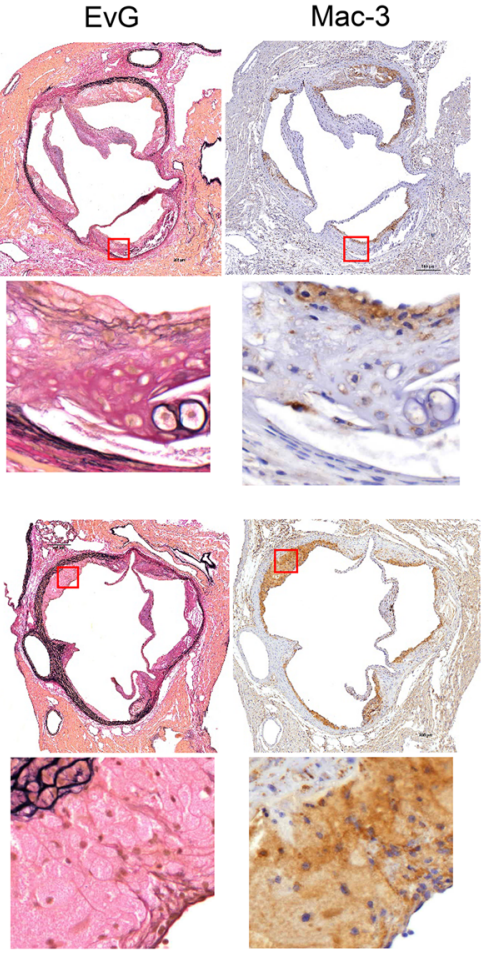

Fig. 1 Prevention in apoE KO mice. Values are expressed as mean \pm SEM (a, b) or mean with scatter plots $(\mathbf{c}-\mathbf{e})(n=16)$. (A) Body weight change. b Food intake. $\mathbf{c}$ Lesion area in en face analysis. ${ }^{* *} P<0.01$ as compared with control. $\mathbf{d}$ Cholesterol content in aorta. ${ }^{* *} P<0.01$ as compared with control. e Lesion area of aortic root $(n=5-7)$. ${ }^{*} P<0.05$ as compared with control. $\mathbf{f}$ Aorta with Sudan IV staining. $\mathbf{g}$ Histological features using serial cross sections subjected to HE, EvG and anti Mac-3 antigen. In the EvG staining, elastic fibers and nuclei appear in dark purple, collagen fibers in red, smooth muscle and cytosol between internal and external laminae in yellow 
a

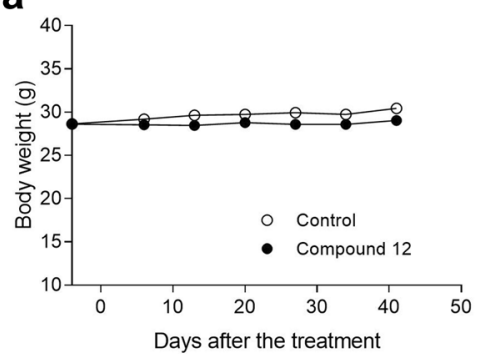

b

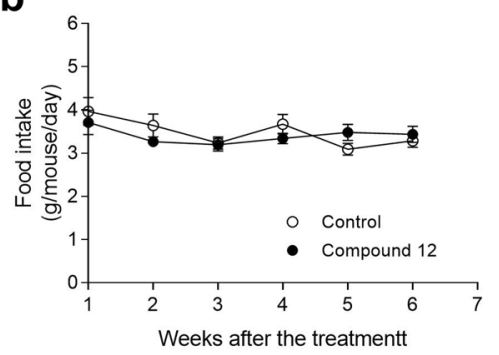

C

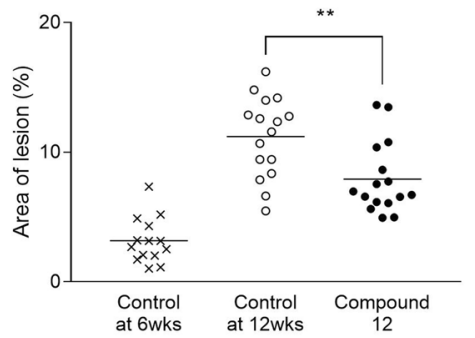

d

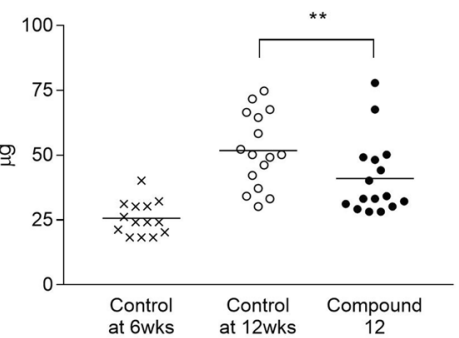

e

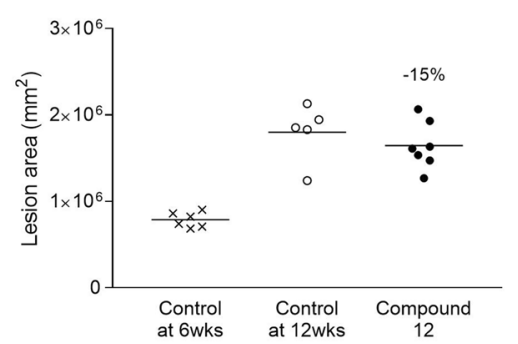

Fig. 2 Therapeutic intervention in apoE KO mice. Values are expressed as mean \pm SEM $(\mathbf{a}, \mathbf{b})$ or mean with scatter plots $(\mathbf{c}-\mathbf{e})(\mathrm{n}=16)$. $\mathbf{a}$ Body weight change. $\mathbf{b}$ Food intake. $\mathbf{c}$ Lesion area in en face analysis. ${ }^{* *} ; P<0.01$ as compared with control. $\mathbf{d}$ Cholesterol content in aorta. ${ }^{*} P<0.05$ as compared with control. e Lesion area of aortic root $(n=5-7)$. ${ }^{*} P<0.05$ as compared with control

a

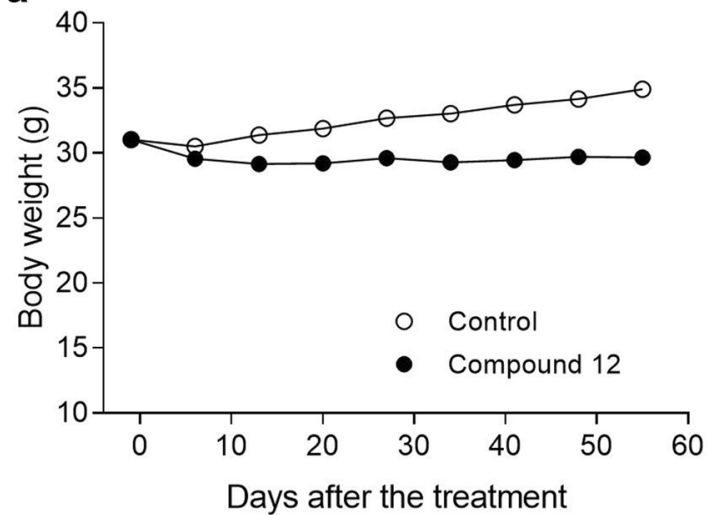

C

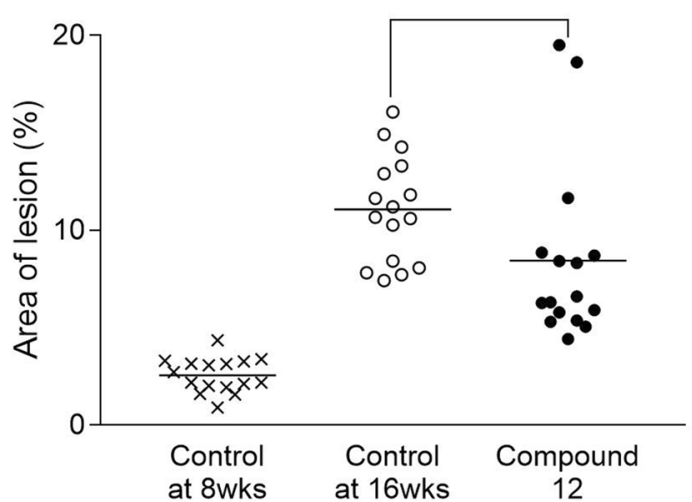

b

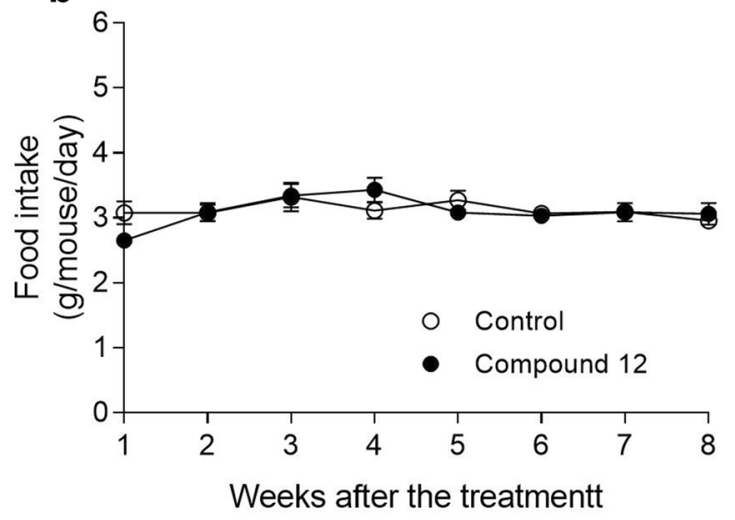

d

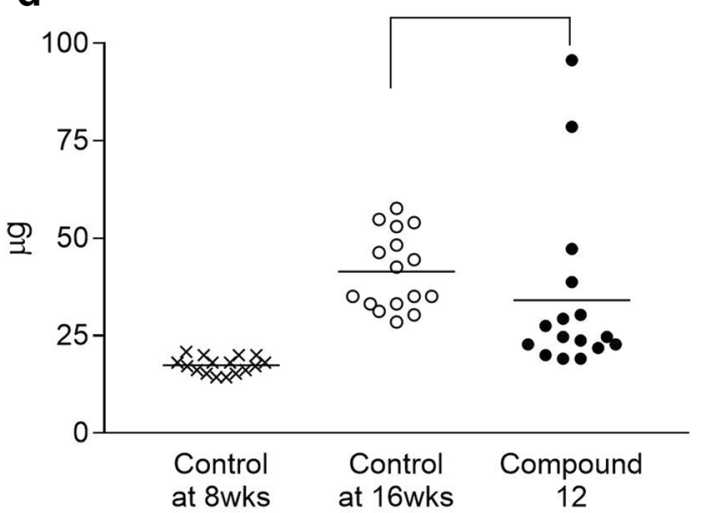

Fig. 3 Therapeutic intervention in LDLr KO mice. Values are expressed as mean $\pm \operatorname{SEM}(\mathbf{a}, \mathbf{b})$ or mean with scatter plots $(\mathbf{c}, \mathbf{d})(\mathrm{n}=16)$. (A) Body weight change. b Food intake. $\mathbf{c}$ Lesion area in en face analysis. ${ }^{*} P<0.05$ as compared with control. $\mathbf{d}$ Cholesterol content in aorta. ${ }^{*} P<0.01$ as compared with control 
pharmacodynamic and functional assays will be required to elucidate the mechanism of action of FAK inhibition as well as to reveal a pathological role of FAK in atherosclerosis.

\section{Additional files}

Additional file 1: Figure S1. The structure of Compound 12. Chemical name: 7-[5-Chloro-2-(2,4-dimethoxy-phenylamino)-pyrimidin-4-ylamino]2-methyl-4-[4-(4-methyl-piperazin-1-yl)-piperidin-1-yl]-2,3-dihydroisoindol-1-one.

Additional file 2: Table S1. $\mathrm{IC}_{50}$ values; Kinase inhibition selectivity of compound 12

Additional file 3: Figure S2. Overview of efficacy trials. (A) Prevention in apoE KO mice, (B) therapeutic intervention in apoE KO mice $(6+6$ weeks) and LDLr KO mice ( $8+8$ weeks).

Additional file 4: Figure S3. Atherosclerotic lesion area analysis. Atherosclerotic lesion area was evaluated in the aorta by en face analysis (A) and in aortic root by cross-sectional analysis (B).

Additional file 5: Table S2. Clinical biochemistry markers; Data expressed as mean \pm SEM.

\section{Abbreviations}

ALB: albumin; ALP: alkaline phosphatase; ALT: alanine aminotransferase; AOWA: area of whole aorta; apoE: apolipoprotein E; AST: aspartate aminotransferase; ATP: adenosine triphosphate; BUN: blood urea nitrogen; CPK: creatine phosphokinase; CRE: creatinine; EvG: Elastica van Gieson; FAK: focal adhesion kinase; Glc: glucose; HC: high cholesterol; HE: hematoxylin eosin; HF: high fat; LDH: lactate dehydrogenase; LDLr: low-density lipoprotein receptor; SMA: smooth muscle actin; TAOL: total area of the plaque lesion; T-BIL: total bilirubin; T-Chol: total cholesterol; TG: triglyceride.

\section{Authors' contributions}

TY, TK, NI, MK and SH contributed to planning the experiments. TY, TK, NI and MK conducted the experiments and analyses, and SH drafted the manuscript. All other authors edited and revised the manuscript. All authors read and approved the final manuscript.

\section{Author details}

${ }^{1}$ Novartis Institutes for BioMedical Research, Novartis Pharma K.K, Tsukuba, Ibaraki, Japan. ${ }^{2}$ Novartis Pharma AG, Basel, Switzerland.

\section{Acknowledgements}

We thank Novartis Institutes for BioMedical Research for their support.

\section{Competing interests}

All authors are or were employees of Novartis Pharma at the time of the work was carried out.

\section{Availability of data and materials}

The datasets used in the current study are available from the corresponding author by request.

\section{Consent for publication}

Not applicable.

\section{Ethics approval and consent to participate}

Animal experimental procedures in this study were in compliant with the regulations of Animal Welfare Committee in Novartis Institutes for BioMedical Research Tsukuba.

Funding

Not applicable.

\section{Publisher's Note}

Springer Nature remains neutral with regard to jurisdictional claims in published maps and institutional affiliations.

Received: 1 February 2019 Accepted: 25 March 2019

Published online: 02 April 2019

\section{References}

1. Schlaepfer DD, Hauck CR, Sieg DJ. Signaling through focal adhesion kinase. Prog Biophys Mol Biol. 1999;71:435-78.

2. Hecker TP, Gladson CL. Focal adhesion kinase in cancer. Front Biosci. 2003;8:s705-14

3. Gabarra-Niecko V, Schaller MD, Dunty JM. FAK regulates biological processes important for the pathogenesis of cancer. Cancer Metastasis Rev. 2003;22:359-74.

4. Rose DM, Liu S, Woodside DG, et al. Paxillin binding to the alpha 4 integrin subunit stimulates LFA-1 (integrin alpha L beta 2)-dependent $T$ cell migration by augmenting the activation of focal adhesion kinase/prolinerich tyrosine kinase-2. J Immunol. 2003;170:5912-8.

5. Sundberg $\sqcup$, Galante $L M$, Bill HM, et al. An endogenous inhibitor of focal adhesion kinase blocks Rac1/JNK but not Ras/ERK-dependent signaling in vascular smooth muscle cells. J Biol Chem. 2003:278:29783-91.

6. Kawahara E, Miyake T, Matsuura N, et al. Discovery of potent and selective focal adhesion kinase inhibitors. Eur J Cancer. 2008;6(Suppl):43. https:// doi.org/10.1016/s1359-6349(08)72065-2.

7. Kawakami A, Yoshida M. Remnant lipoproteins and atherogenesis. J Atheroscler Thromb. 2005;12:73-6.

8. Chen Y, Cao J, Zhao Q, et al. Silencing MR-1 attenuates atherosclerosis in ApoE-/- mice induced by angiotensin II through FAK-Akt-mTOR-NFkappaB signaling pathway. Korean J Physiol Pharmacol. 2018;22:127-34.

9. Yurdagul A Jr, Sulzmaier FJ, Chen XL, et al. Oxidized LDL induces FAKdependent RSK signaling to drive NF-KB activation and VCAM-1 expression. J Cell Sci. 2016:129:1580-91.

Ready to submit your research? Choose BMC and benefit from:

- fast, convenient online submission

- thorough peer review by experienced researchers in your field

- rapid publication on acceptance

- support for research data, including large and complex data types

- gold Open Access which fosters wider collaboration and increased citations

- maximum visibility for your research: over 100M website views per year

At BMC, research is always in progress.

Learn more biomedcentral.com/submissions 